\title{
SELF-DUAL CONFIGURATIONS AND THEIR LEVI GRAPHS
}

\author{
RAFAEL ARTZY
}

Introduction. By a configuration we shall mean a set of points and straight lines (or planes) between which certain well-determined incidences exist. These configurations are not necessarily regular, that is, we shall not suppose every line (plane) to be incident with the same number of configuration points, and dually, different points of the configuration need not be incident with the same number of configuration lines (planes). The present paper will treat only self-dual configurations. (In this section and the next, we shall speak of configurations of points and lines only, but the reader may put "plane" instead of "line.")

As Coxeter [1] has pointed out, configurations have been described by graphs of two kinds: the Menger graph which has the disadvantage of not determining uniquely the configuration which it describes, and the Levi graph which in general is rather cumbersome because it has one node for each point and another for each line of the configuration. Thus the Levi graph of a self-dual configuration of $n$ points has $2 n$ nodes. In order to overcome this inconvenience of the Levi graph we propose here to identify abstractly the node of every point with the node of its dually corresponding line, and thus to construct a reduced Levi graph (in short RLG) which has only $n$ nodes and which preserves the advantages of the Levi graph: one RLG cannot describe two distinct configurations. On the other hand the RLG will be shown to have the disadvantage that one configuration may have several nonisomorphic RLG's. In RLG's a node may be joined to itself, thus forming a loop.

We shall see that the RLG permits sometimes a convenient discussion of self-dual specializations of self-dual configurations, in addition to its uses as a simpler form of the Levi graph.

1. The RLG. The Levi graph [3, p. 5] of a configuration has just one node representing each point of the configuration, and just one node representing each of its lines. A point-node is joined to a linenode whenever the represented point and line are incident with each other. In a self-dual configuration every reciprocity determines a oneto-one correspondence between the lines and the points. By identi-

Presented to the Society, September 3, 1954; received by the editors January 10, 1955 and, in revised form, May 20, 1955. 
fying abstractly every point-node of the Levi graph of such a configuration with its corresponding line-node we get an RLG. The reciprocity $r$ implies that if the point $P$ lies on the line $d$, the point $r(d)$ is also incident with the line $r(P)$. In the RLG, $P$ and $r(P)$ are represented by one node which is joined to the node which stands for $d$ and $r(d)$. When in the configuration $P$ and $r(P)$ are incident, the node of $P$ and $r(P)$ in the RLG has a loop.

Since each reciprocity determines an RLG, a configuration may have several nonisomorphic RLG's. A simple example is the triangle which has two forms of RLG: the first is a triangle without loops; the other has two nodes with loops, joined to the third node, but without a branch joining them.

On the other hand, a given RLG allows the construction of a unique Levi graph. Since two nonisomorphic configurations cannot have the same Levi graph, a configuration is uniquely determined by an RLG.

2. Examples. A few examples of important self-dual configurations follow:

2.1. Pappus's configuration (the lettering according to $[2, \mathrm{p} .40$, fig. 4.3A and $\mathrm{B}]$ ) has only one form of RLG. The points represented by the loop-nodes of the RLG (see figure 2.1) are the vertices and sides of any triangle of the configuration, as, for example, $A^{\prime}, a^{\prime}$; $B^{\prime}, b^{\prime} ; N, n$; the position of the remaining nodes is uniquely determined by the selection of this triangle. Since there are 18 triangles in the configuration, and 6 automorphisms in the RLG, we get 108 as the group order of the configuration, excluding the reciprocities.

2.2. ${ }^{1}$ Möbius-Kantor's 8 has two different forms of RLG: one with four loops (see figure 2.2), and another with two loops.

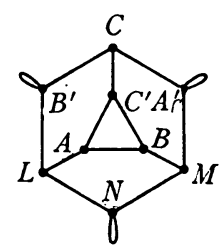

FIG. 2.1

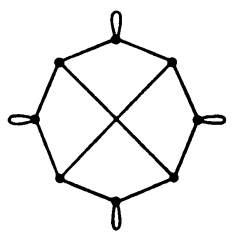

FIG. 2.2

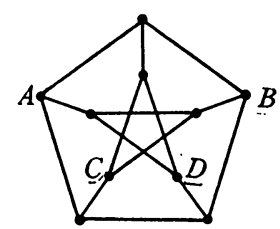

Fig. 3

2.3. ${ }^{1}$ Möbius's 84 is considered as a configuration of points and planes. There are three distinct forms of RLG. One of them has the 8 vertices of a cube as nodes, each with a loop, and the edges of the cube as branches. In another form of RLG the 8 nodes correspond to the vertices of two tetrahedra, $A B C D, E F G H$ (without any loops)

\footnotetext{
${ }^{1}$ The nomenclature of the configurations is that of the comprehensive paper by Coxeter [1].
} 
and the 16 branches to the 12 edges of the tetrahedra and to the connecting lines $A E, B F, C G, D H$.

\section{Self-dual specializations of configurations.}

3.0. The following specialization of a self-dual configuration yields again a self-dual configuration: A point $P$ of the configuration is made to be incident with a line (or plane) $d$ of the configuration, and the point $r(d)$ becomes incident with the line (plane) $r(P)$. In the RLG this specialization is performed by the addition of a branch joining the nodes which represent $P$ and $d$. We get a special case of this procedure when $d=r(P)$. In the RLG this signifies that a loop is added to an existing node which stands for $P$.

3.1. As an example we consider the Desargues $10_{3}$ configuration. Its RLG can have several forms; we shall deal mainly with one of them which corresponds to the unique von Staudt polarity between any pair of Desargues triangles (cf. [2, p. 65]). The resulting RLG is the Petersen graph, without any loops (figure 3 ).

It is interesting that the Petersen graph has been shown by Kagno [4] to be also the complement of the Menger graph of the Desargues configuration, thus implying the isomorphism of the groups of both graphs.

A further remark: The Petersen graph is the most symmetrical form of RLG of the Desargues configuration; there is another form with loops, and one regular form without loops, but containing two triangles. Another case in which a configuration has a single privileged reciprocity is that of Cox's $\left(2^{d-1}\right)_{d}$ for any odd value of $d[1$, p. 448]; the corresponding RLG consists of the vertices and edges of $\gamma_{d} / 2$, or $\gamma_{d-1}$ with opposite vertices joined.

3.2. The addition of a loop at any one of the nodes of the Petersen graph yields the RLG of the "Small Desargues configuration" where the perspective center of the two triangles lies on the "Desargues line" which passes through the points of intersection of the three pairs of corresponding sides of the triangles.

3.3. The addition of loops at three nodes of the Petersen graph which are not joined to one another, nor to a common further node (as, for instance, $A, B, C$ ), yields another specialization of the Desargues configuration: the vertices of one of the triangles lie on the sides of the other triangle.

3.4. The addition of loops at four nodes which fulfill the same conditions as specified in 3.3 (for instance, $A, B, C, D$ ) results in a specialization which combines those of 3.2 and 3.3. The configuration thus produced is not embeddable in the usual projective plane, but it is embeddable in $P G(2,3)$. The group of the RLG is the octa- 
hedral group; in fact the RLG can be drawn as a tetrahedron $A B C D$ with loops at the vertices and extra nodes on the six edges, the nodes on opposite edges being joined.

4. Finite geometries. Three examples of the RLG's of finite geometries will be mentioned:

4.1. $P G(2,2)$ yields a unique RLG having seven nodes $(0, \cdots, 6)$ with $i$ and $j$ joined whenever $i+j \equiv 0,1$ or $3(\bmod 7)$. There are, accordingly, loops at 0,4 , and 5 .

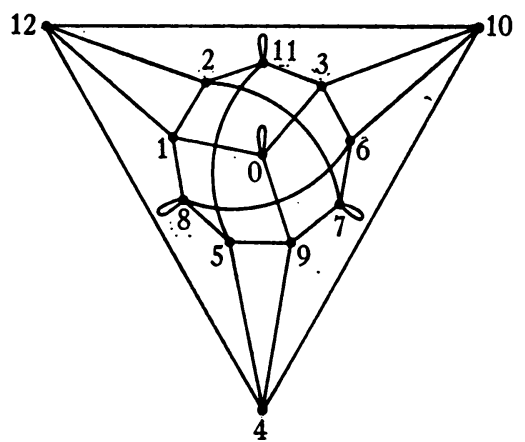

FIG. 4.2

4.2. $P G(2,3)$ has only one form of RLG, with loops at four nodes $(0,7,8,11$ in figure 4.2$)$. This graph is seen to be so arranged that nodes $i$ and $j$ are joined whenever

$$
i+j \equiv 0,1,3 \text { or } 9(\bmod 13) \text {. }
$$

Its group is the octahedral group.

The consistency of the configurations of Pappus, Möbius-Kantor and of the special Desargues configuration 3.4 with $P G(2,3)$ can be easily seen as follows:

By deleting the nodes $4,10,12$ and all the branches which connect with them, we get the RLG of 3.4 .

By deleting 4, 5, 9, 10, 12 and all the branches which connect with them, we get the RLG of 2.2.

By deleting the nodes $0,1,3,9$ and all the branches which connect with them, including the loop at 0 , we get the RLG of 2.1 .

4.3. $P G(3,2)$, considered as a configuration of points and planes, is represented by an RLG consisting of the RLG of 2.3 (two tetrahedra), without any loops, and seven additional nodes each of which has a loop. These seven nodes $J, K, L, M, N, P, Q$ are joined to one another and to the nodes $A, B, C, D, E, F, G, H$ as in the schematic figure 4.3. 
The embeddability of Möbius's $8_{4}$ in $P G(3,2)$ is now evident.

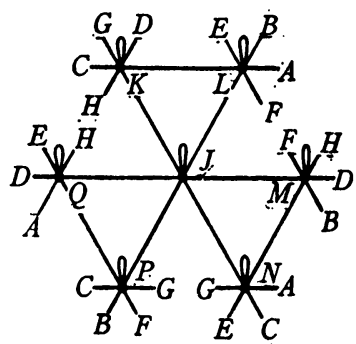

FIG. 4.3

Added in proof. Following Pickert's classification of the configurations of degenerate Desargues theorems [5, pp. 86-89] we are able to add two further specializations:

(a) By adding loops at two nodes which are not joined to each other (as, for instance, $A$ and $B$ ) we get the configuration of the doubly degenerate Desargues theorem where, in addition to the specialization of the small Desargues configuration, the vertex of one triangle lies on a side of the other triangle without, however, coinciding with one of its vertices.

(b) We get the configuration of a triply degenerate Desargues theorem (besides that of 3.3) by adding loops at three nodes of the Petersen graph which are not connected to each other but all of which are joined to one further node. Here we have a special Small Desargues configuration in which two vertices of one triangle are incident with the respective opposite sides of the other triangle. This configuration is known $[5$, p. 88$]$ to be embeddable in desarguesian planes only when their coordinate division ring has the characteristic two.

\section{REFERENCES}

1. H. S. M. Coxeter, Self-dual configurations and regular graphs, Bull. Amer. Math. Soc. vol. 56 (1950) pp. 413-455.

2. - The real projective plane, New York, 1949.

3. F. W. Levi, Finite geometrical systems, Calcutta, 1942.

4. I. N. Kagno, Desargues' and Pappus' graphs and their groups, Amer. J. Math. vol. 69 (1947) pp. 859-862.

5. G. Pickert, Projektive Ebenen, Berlin, 1955.

ISRAEL InSTITUTE OF TECHNOLOGY 